УДК 519.216, 519.866.2

\title{
ФЕЛЛЕРОВСКОЕ ПЕРЕХОДНОЕ ЯДРО С НОСИТЕЛЯМИ МЕР, ЗАДАННЫМИ МНОГОЗНАЧНЫМ ОТОБРАЖЕНИЕМ ${ }^{1}$
}

\author{
С. Н. Смирнов
}

\begin{abstract}
Пусть $X$ - топологическое пространство и $Y$ - сепарабельное метрическое пространство, снабженные борелевскими $\sigma$-алгебрами $\mathcal{B}_{X}$ и $\mathcal{B}_{Y}$ соответственно; $P(x, B)$ - стохастическое переходное ядро (т. е. отображение $x \mapsto P(x, B)$ измеримо для всех $B \in \mathcal{B}_{Y}$ и отображение $B \mapsto P(x, B)$ - вероятностная мера для всех $x \in X) ; \operatorname{supp}(P(x, \cdot))$ - топологический носитель меры $B \mapsto P(x, B)$. Если переходное ядро $P(x, B)$ удовлетворяет феллеровскому свойству (т. е. отображение $x \mapsto P(x, \cdot)$ непрерывно по отношению к слабой топологии на пространстве вероятностных мер), тогда многозначное отображение $x \mapsto \operatorname{supp}(P(x, \cdot))$ полунепрерывно снизу. Обратно, пусть задано многозначное отображение $x \mapsto S(x)$, где $x \in X$ и $S(x)-$ непустое замкнутое подмножество польского пространства $Y$. Если $x \mapsto S(x)$ полунепрерывно снизу, то при достаточно общих предположениях относительно топологического пространства $X$ существует феллеровское переходное ядро, такое что $\operatorname{supp}(P(x, \cdot))=S(x)$ для всех $x \in X$.
\end{abstract}

Ключевые слова: феллеровское свойство, переходное ядро, топологический носитель меры, полунепрерывное снизу многозначное отображение, непрерывная ветвь (селектор).

S. N. Smirnov. A Feller transition kernel with measure supports given by a set-valued mapping.

Assume that $X$ is a topological space and $Y$ is a separable metric space. Let these spaces be equipped with Borel $\sigma$-algebras $\mathcal{B}_{X}$ and $\mathcal{B}_{Y}$, respectively. Suppose that $P(x, B)$ is a stochastic transition kernel; i.e., the mapping $x \mapsto P(x, B)$ is measurable for all $B \in \mathcal{B}_{Y}$ and the mapping $B \mapsto P(x, B)$ is a probability measure for any $x \in X$. Denote by $\operatorname{supp}(P(x, \cdot))$ the topological support of the measure $B \mapsto P(x, B)$. If the transition kernel $P(x, B)$ satisfies the Feller property, i.e., the mapping $x \mapsto P(x, \cdot)$ is continuous in the weak topology on the space of probability measures, then the set-valued mapping $x \mapsto \operatorname{supp}(P(x, \cdot))$ is lower semicontinuous. Conversely, consider a set-valued mapping $x \mapsto S(x)$, where $x \in X$ and $S(x)$ is a nonempty closed subset of a Polish space $Y$. If $x \mapsto S(x)$ is lower semicontinuous, then, under some general assumptions on the space $X$, there exists a Feller transition kernel such that $\operatorname{supp}(P(x, \cdot))=S(x)$ for all $x \in X$.

Keywords: Feller property, transition kernel, topological support of a measure, lower semicontinuous setvalued mapping, continuous branch (selection).

MSC: 60J35, 91B25

DOI: $10.21538 / 0134-4889-2019-25-1-219-228$

\section{Введение}

Феллеровское свойство, говоря неформальным языком, означает, что стохастическая динамика изучаемого объекта описывается посредством условных вероятностей при известной предыстории процесса, представляющих собой "гладкую" функцию ${ }^{2}$ от наблюдаемой истории процесса (в частности, от его текущего состояния в случае марковского процесса). Это свойство является естественным в физическом или социальном моделировании. Кроме того, марковские процессы, удовлетворяющие свойству Феллера, образуют важный класс случайных процессов (см., например, [1]).

\footnotetext{
${ }^{1}$ Исследование выполнено в рамках проведения научно-исследовательских работ на факультете ВМК МГУ имени М.В. Ломоносова по теме "Методы оптимизации в задачах управления для сложных систем в условиях реально доступной информации" (№ госрегистрации АААА-А16-116021110324-8).

${ }^{2}$ Речь идет о непрерывной зависимости; строгое определение феллеровского свойства приведено далее.
} 
Интересно, что настоящая работа возникла в финансовом контексте, а именно при изучении проблемы суперхеджирования для дискретного времени в гарантированной детерминистской постановке задачи, когда возможное движение (т. е. приращение) ценового вектора за один шаг описывается многозначным отображением с непустыми компактными значениями. В этом контексте естественным образом возникает задача управления в условиях неопределенности ${ }^{3}$. Данный подход можно также интерпретировать как динамическую антагонистическую игру между двумя "противниками". Первый из них - "хеджер", использующий чистые стратегии управления портфелем с целью гарантированного покрытия обусловленных обязательств по опциону. Второй - "рынок", использующий смешанные стратегии поведения цен, которые описываются посредством конструкции [3], т. е. при помощи построения вероятностной меры на основе заданных переходных ядер; последние можно интерпретировать как условные вероятности при известной предыстории этого случайного процесса. Мы считаем, что упомянутое выше многозначное отображение вряд ли можно рассматривать как реалистичную (многозначную) динамику ценового вектора, если не найдется смешанных стратегий, непрерывно зависящих от наблюдаемой истории цен, с соответствующими носителями, зависящими от предыстории цен. Это объясняет прикладной интерес к проблеме, представленной в данной статье.

Основным результатом работы является следующее утверждение: существование феллеровского переходного ядра с носителями условных вероятностей, содержащихся в множествах, заданных некоторым многозначным отображением, эквивалентно полунепрерывности снизу этого многозначного отображения при некоторых естественных предположениях о топологических пространствах. В качестве математического инструментария мы используем классические результаты Майкла [4] о непрерывных ветвях (селекторах) многозначных отображений.

\section{1. Вспомогательные результаты}

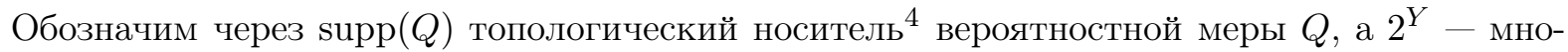
жество всех подмножеств $Y$.

Доказательство следующего простого результата можно найти, например, в [5, лемма 4.3].

Лемма 1. Пусть $Y$ - сепарабельное метрическое пространство. Тогда многозначное отображение $Q \mapsto \operatorname{supp}(Q)$ является полунепрерывным снизу ${ }^{5}$ для слабой топологии ${ }^{6}$ на пространстве вероятностных мер $\mathcal{P}(Y)$, заданных на борелевской б-алгебре $\mathcal{B}_{Y}$.

Для заданной пары измеримых пространств $\left(X, \mathcal{A}_{X}\right)$ и $\left(Y, \mathcal{A}_{Y}\right)$ стохастическое переходное ядро $P$ определяется как числовая функция $(x, A) \mapsto P(x, A)$, такая что функции $x \mapsto P(x, A)$

\footnotetext{
${ }^{3}$ Гарантированный подход для общего случая задачи управления в условиях неопределенности изложен в книге А. Б. Куржанского [2].

${ }^{4}$ Топологический носитель меры на топологическом пространстве с борелевской $\sigma$-алгеброй определяют как множество точек, любая окрестность которых имеет положительную меру; такое множество замкнуто. Однако без дополнительных предположений о топологическом пространстве нельзя гарантировать, что дополнение носителя (представимое в виде объединения открытых множеств нулевой меры) будет иметь нулевую меру; наличие счетной базы снимает проблему. Отметим, что топологический носитель может быть пустым (см., например, $[6$, предложение 3.5, гл. 1] - принадлежащий Дьедонне пример вероятностной меры, заданной на борелевской $\sigma$-алгебре компактного хаусдорфова пространства, с пустым носителем (при этом мера не является регулярной)).

${ }^{5}$ Многозначное отображение $F: X \mapsto 2^{Y} \backslash\{\varnothing\}$ полунепрерывно снизу, если для любого открытого множества $G \subseteq Y$ множество $\{x \in X: F(x) \cap G \neq \varnothing\}$ является открытым (см., например, [7, определение 2.3 , замечание 2.4 и эквивалентные формулировки в предложении 2.6$]$ ).

${ }^{6}$ Слабейшая топология, для которой функционалы $Q \mapsto \int f d Q$ непрерывны для любой ограниченной непрерывной функции $f: Y \mapsto \mathbb{R}$.
} 
являются ${ }^{7} \mathcal{A}_{X}$-измеримыми для всех $A \in \mathcal{A}_{Y}$, а функции $A \mapsto P(x, A)$ являются нормированными (вероятностными) мерами на $\mathcal{A}_{Y}$ для всех $x \in X$. Поскольку далее рассматриваются только стохастические переходные ядра, будем называть их просто "переходные ядра", опуская слово "стохастические". Переходное ядро $Q=Q(x, B)$ удовлетворяет феллеровскому свойству, если отображение $x \mapsto Q(x, \cdot)$ непрерывно (по отношению к слабой топологии на пространстве вероятностных мер).

Предложение 1. Пусть $X$ - топологическое пространство и $Y$ - метрическое пространство с заданными на них борелевскими б-алгебрами $\mathcal{B}_{X}$ и $\mathcal{B}_{Y}$ соответственно. Если отображение $x \mapsto Q(x, \cdot) \in \mathcal{P}(Y), x \in X$, непрерывно для слабой топологии на $\mathcal{P}(Y)$, то $Q=Q(x, B), x \in X, B \in \mathcal{B}_{Y}$ является переходным ядром, т.е. для любого $B \in \mathcal{B}_{Y}$ числовая функиия $x \mapsto Q(x, B)$ является $\mathcal{B}_{X}$-измеримой.

Д о к а з а т е л ь с т в о. Обозначим через $\mathcal{D}$ класс всех множеств $B \in \mathcal{B}_{Y}$, таких что функция $x \mapsto Q(x, B)$ является $\mathcal{B}_{X}$-измеримой. Нетрудно видеть, что $\mathcal{D}$ образует систему Дынкина (см. например, [10, определение 4.1.1]), т. е.

1) $Y \in \mathcal{D}$ (так как функция-константа измерима);

2) если $A, B \in \mathcal{D}$ и $A \subseteq B$, то $A \backslash B \in \mathcal{D}$ (так как разность измеримых функций измерима);

3) если $A_{1}, A_{2}, \ldots \in \mathcal{D}$ и $A_{1} \subseteq A_{2} \subseteq \ldots$, то $\cup_{i=1}^{\infty} A_{i} \in \mathcal{D}$ (так как предел измеримых функций измерим).

Заметим, что топология $\mathcal{J}_{Y}$ на $Y$ содержится в $\mathcal{D}$, поскольку в силу непрерывности отображения $x \mapsto Q(x, \cdot)$ для открытого множества $G \subseteq Y$ функция $x \mapsto Q(x, G)$ полунепрерывна снизу: по теореме А. Д. Александрова ${ }^{8}$ для сходящейся направленности $x_{\alpha} \rightarrow x$ направленность мер $Q\left(x_{\alpha}, \cdot\right)$ удовлетворяет свойству $\liminf Q\left(x_{\alpha}, G\right) \geq Q(x, G)$ для любого $G \in \mathcal{J}_{Y}$. Полунепрерывная снизу функция $x \mapsto Q(x, G)$ является $\mathcal{B}_{X}$-измеримой, поскольку множество $\{x \in X: Q(x, G)>a\}$ открыто, а значит принадлежит $\mathcal{B}_{X}$ для любого $a \in \mathbb{R}$ (см., например, $\left[12\right.$, следствие из леммы $1, \S 4$, гл. 2]). Поскольку топология $\mathcal{J}_{Y}$ замкнута относительно конечных пересечений, то применима теорема о системах Дынкина (см. например, $[10$, теорема 4.1.2]), в соответствии с которой $\mathcal{D}$ содержит минимальную $\sigma$-алгебру, содержащую $\mathcal{J}_{Y}$, т. е. борелевскую $\sigma$-алгебру $\mathcal{B}_{Y}$. Тем самым для любого $B \in \mathcal{B}_{Y}$ функция $x \mapsto Q(x, B)$ является $\mathcal{B}_{X}$-измеримой.

Предложение 2. Пусть $X$ - топологическое пространство и $Y$ - сепарабельное метрическое пространство ${ }^{9}$ с заданными на них борелевскими $\sigma$-алгебрами $\mathcal{B}_{X}$ и $\mathcal{B}_{Y}$ соответственно. Если переходное ядро $Q=Q(x, B), x \in X, B \in \mathcal{B}_{Y}$, обладает феллеровским свойством, то многозначное отображение $x \mapsto \operatorname{supp}(Q(x, \cdot))$ является полунепрерывным снизу.

Д о к а з а т е л ь с т в о. Обозначим $\varphi(x)=Q(x, \cdot)$; (однозначное) отображение $\varphi: X \mapsto$ $\mathcal{P}(Y)$ (слабо) непрерывно в силу предположения о феллеровости $Q$. Очевидно, что

$$
\{x \in X: \operatorname{supp}(\varphi(x)) \cap G \neq \varnothing\}=\varphi^{-1}\left(\mathcal{M}_{G}\right)
$$

где

$$
\mathcal{M}_{G}=\{Q \in \mathcal{P}(Y): \operatorname{supp}(Q) \cap G \neq \varnothing\}
$$

\footnotetext{
${ }^{7}$ На $\mathbb{R}$ задается борелевская $\sigma$-алгебра $\mathcal{B}_{\mathbb{R}}$.

${ }^{8}$ Теорему естественно так называть, так как первый результат такого типа был опубликован в работе $[9$, теорема $2, \S 16]$. В действительности, нужное нам утверждение содержится в книге [11, т. 2], теорема 8.2.3 и следствие 8.2.4. Кстати, доказательство леммы 1 также опирается на теорему А. Д. Александрова.

${ }^{9}$ Слабая топология на $\mathcal{P}(Y)$ для сепарабельного пространства $Y$ метризуема (см., например, [8, теорема 5 из дополнения III]), поэтому (учитывая, что мера на сепарабельном пространстве сама сепарабельна) можно рассматривать последовательности, а не направленности мер.
} 
в силу леммы 1 для открытого $G$ множество $\mathcal{M}_{G}$ открыто, а его прообраз открыт в силу непрерывности $\varphi$.

Пусть $(Y, \rho)$ - сепарабельное метрическое пространство. Рассмотрим линейное пространство $B L(Y)$ ограниченных липшицевых функций $f: Y \mapsto \mathbb{R}$ с нормой ${ }^{10}$

$$
\begin{gathered}
\|f\|_{B L}=\|f\|_{L}+\|f\|_{\infty}, \\
\|f\|_{\infty}=\sup \{|f(y)|: y \in Y\}, \\
\|f\|_{L}=\sup \left\{\frac{\left|f(y)-f\left(y^{\prime}\right)\right|}{\rho\left(y, y^{\prime}\right)}: y, y^{\prime} \in Y, y \neq y^{\prime}\right\},
\end{gathered}
$$

а также нормированное векторное пространство $\mathcal{M}(Y)$ зарядов (знакопеременных мер) на пространстве $Y$ с борелевской $\sigma$-алгеброй $\mathcal{B}_{Y}$, снабженной нормой Дадли (Dudley) ${ }^{11}$

$$
\|\mu\|_{D}=\sup \left\{\left|\int f d \mu\right|:\|f\|_{B L} \leq 1\right\} .
$$

Множество всех нормированных (вероятностных) мер $\mathcal{P}(Y) \subseteq \mathcal{M}(Y)$ будет замкнутым выпуклым подмножеством $\mathcal{M}(Y)$ с метрикой $\rho_{D}$, порожденной нормой $\|\cdot\|_{D}$, т. е. $\rho_{D}\left(\pi_{1}, \pi_{2}\right)=$ $\left\|\pi_{1}-\pi_{2}\right\|_{D}$

Лемма 2. Пусть $\left(Y, \mathcal{B}_{Y}\right)$ - сепарабельное метрическое пространство с борелевской $\sigma$-алгеброй. Тогда любую вероятностную меру $Q$ на $\left(Y, \mathcal{B}_{Y}\right)$ можно приблизить в метрике Дадли вероятностными мерами с конечными носителями, содержащимися в носителе $Q$, в том смысле, что найдется слабо сходящаяся последовательность вероятностных мер $Q_{n}$, таких чmo $\operatorname{supp}\left(Q_{n}\right) \subseteq \operatorname{supp}(Q)$ u $\left\|Q_{n}-Q\right\|_{D} \rightarrow 0$.

Д о к а з а т е л ь с т в о. Фиксируем произвольное $\varepsilon>0$ и $Q \in \mathcal{P}(Y)$. Если $Y$ конечно, то доказывать нечего, поэтому предположим, что $Y$ бесконечно. Пусть $C=\left\{x_{1}, x_{2}, \ldots\right\}-$ счетное всюду плотное в $Y$ множество. Обозначим $S=\operatorname{supp}(Q)$ и $B_{\varepsilon}(x)-$ открытый шар радиуса $\varepsilon$ с центром $x$. Рекуррентно построим последовательность множеств $A_{1}, A_{2}, \ldots$, попарно непересекающихся и таких что ${ }^{12} \sum_{n=1}^{\infty} A_{n} \supseteq S$; в частности, $Q\left(\sum_{n=1}^{\infty} A_{n}\right)=1$. Положим $A_{1}=B_{\varepsilon}\left(x_{1}\right)$, $A_{n+1}=B_{\varepsilon}\left(x_{n+1}\right) \backslash \sum_{i=1}^{n} A_{i}, n=1,2, \ldots$ Обозначим $J=\left\{k \geq 1: A_{k} \cap S \neq \varnothing\right\}$. Если $n \notin J$, то $Q\left(A_{n}\right)=0$, поэтому $\sum_{k \in J} Q\left(A_{k}\right)=1$. Положим ${ }^{13} Q_{\varepsilon}=\sum_{k \in J} q_{k} \delta_{x_{k}^{*}}, q_{k}=Q\left(A_{k}\right)$, где для $k \in J$ точка $x_{k}^{*}$ произвольно выбрана в $A_{k} \cap S \neq \varnothing$. Заметим, что по построению $A_{k} \subseteq B_{\varepsilon}\left(x_{k}\right)$ и для $k \in J$ множество $A_{k}$ имеет диаметр, не превосходящий $2 \varepsilon$. Поэтому для произвольной числовой функции $f$, такой что $\|f\|_{B L} \leq 1$,

$$
\left|\int f d Q-\int f d Q_{\varepsilon}\right| \leq \sum_{k \in J} \int_{A_{k}}\left|f(x)-f\left(x_{k}^{*}\right)\right| Q(d x) \leq 2 \varepsilon ;
$$

отсюда $\left\|Q-Q_{\varepsilon}\right\|_{D} \leq 2 \varepsilon$. Найдется конечное множество индексов $I \subseteq J$, такое что для его дополнения $I^{c}=J \backslash I$ будет выполняться неравенство ${ }^{14} \delta(I)=\sum_{k \in I^{c}} q_{k} \leq \varepsilon$. Полагая

$$
Q_{\varepsilon}^{\prime}=\sum_{k \in I} q_{k}^{\prime} \delta_{x_{k}^{*}}, \quad q_{k}^{\prime}=\frac{q_{k}}{1-\delta}
$$

\footnotetext{
10Это пространство является банаховым (см., например, [13], где это доказано для эквивалентной нормы $\left.\|f\|_{B L}^{\prime}=\max \left(\|f\|_{L},\|f\|_{\infty}\right)\right)$.

${ }^{11}$ Отметим, что в соответствии с [14, теорема 3.11] нормированное пространство $\mathcal{M}(Y)$ не является полным, если только $Y$ не является равномерно дискретным (когда найдется число $r^{*}>0$, такое что $\rho\left(y_{1}, y_{2}\right) \geq r^{*}$ для всех $\left.y_{1}, y_{2} \in Y\right)$.

${ }^{12}$ Как принято в теории вероятностей, $\sum_{n} A_{n}$ обозначает объединение $\bigcup_{n} A_{n}$ попарно непересекающихся множеств.

13 Здесь $\delta_{x}$ обозначает вероятностную меру, сосредоточенную в точке $x$.

${ }^{14}$ Если $J$ конечно, то можно выбрать $I=J$, тогда $\delta=0$.
} 
получаем $\left\|Q_{\varepsilon}-Q_{\varepsilon}^{\prime}\right\|_{T V}=2 \delta(I) \leq 2 \varepsilon$, где $\|\mu\|_{T V}-$ норма полной вариации конечного заряда $\mu$; поскольку $\|\mu\|_{D} \leq\|\mu\|_{T V}$, то $\left\|Q-Q_{\varepsilon}^{\prime}\right\|_{D} \leq\left\|Q-Q_{\varepsilon}\right\|_{D}+\left\|Q_{\varepsilon}-Q_{\varepsilon}^{\prime}\right\|_{D} \leq 4 \varepsilon$. При этом по построению $\operatorname{supp}\left(Q_{\varepsilon}^{\prime}\right) \subseteq \operatorname{supp}(Q)$. Осталось сослаться на теорему 11.3 .3 из [15], в соответствии с которой для сепарабельного метрического пространства $Y$ слабая сходимость последовательности вероятностных мер равносильна сходимости в метрике Дадли.

Лемма 3. Пусть $F$ - замкнутое множество сепарабельного метрического пространства $Y$. Тогда найдется мера $Q \in \mathcal{P}(Y)$, такая что $\operatorname{supp}(Q)=F$.

Д о к а з а т е л ь с т в о. Случай, когда $F$ конечно, очевиден. Пусть $F$ бесконечно; поскольку (см., например, [16, лемма, разд. $7, \S 4$, гл. III]) $F$ сепарабельно относительно суженной на $F \times F$ метрики, найдется счетное всюду плотное подмножество $C=\left\{x_{1}, x_{2}, \ldots\right\} \subseteq F$. Выберем произвольную последовательность $q_{1}, q_{2}, \ldots$, такую что $q_{n}>0$ для всех $n \geq 1, \sum_{n=1}^{\infty} q_{n}=1$, и положим $Q=\sum_{n=1}^{\infty} q_{n} \delta_{x_{n}}$. Тогда, очевидно, $\operatorname{supp}(Q)=F$.

Предложение 3. Пусть $X$ - топологическое пространство и $Y$ - сепарабельное метрическое пространство, снабженнве борелевскими $\sigma$-алгебрами $\mathcal{B}_{X}$ и $\mathcal{B}_{Y}$ соответственно. Eсли $S: X \mapsto 2^{Y}$ многозначное отображсние, принимающее непустые замкнутые значения, полунепрерывно снизу, то многозначное отображение $\mathcal{N}: X \mapsto \mathcal{P}(Y)$, задаваемое посредством

$$
\mathcal{N}(x)=\{Q \in \mathcal{P}(Y): \operatorname{supp}(Q) \subseteq S(x)\},
$$

принимает непустые замкнутые выпуклые значения и является ${ }^{15}$ h-полунепрерывным снизу для метрики, порожденной нормой Дадли в пространстве $\mathcal{P}(Y)$.

Д о к а з а т е л ь с т в о. Очевидно, $\mathcal{N}(x) \neq \varnothing$ для всех $x \in X$, поскольку $S(x) \neq \varnothing$. Проверим, что для произвольной точки $x_{0} \in X$ и любого $\varepsilon>0$ найдется окрестность $V_{\varepsilon}\left(x_{0}\right)$ точки $x_{0}$, такая что для любого $x \in V_{\varepsilon}\left(x_{0}\right)$ и любой меры $Q_{0} \in \mathcal{N}\left(x_{0}\right)$ существует мера $Q_{\varepsilon}^{x} \in \mathcal{N}(x)$, такая что $\left\|Q_{0}-Q_{\varepsilon}^{x}\right\|_{D}<\varepsilon$. По определению (1.1) для $Q_{0} \in \mathcal{N}\left(x_{0}\right)$ имеем $\operatorname{supp}\left(Q_{0}\right) \subseteq S\left(x_{0}\right)$. В силу леммы 2 найдется мера $Q_{\varepsilon}$ с конечным носителем $\operatorname{supp}\left(Q_{\varepsilon}\right)=\left\{y^{1}, \ldots, y^{m}\right\} \subseteq S\left(x_{0}\right)$ (т. е. представимая в виде $Q_{\varepsilon}=\sum_{i=1}^{m} q_{i} \delta_{y^{i}}$, где $\sum_{i=1}^{m} q_{i}=1, q_{i} \geq 0, i=1, \ldots, m$ ), такая что $\left\|Q_{0}-Q_{\varepsilon}\right\|_{D}<\varepsilon / 2$. В силу предположения о полунепрерывности снизу многозначного отображения ${ }^{16} x \mapsto S(x)$ у выбранной точки $x_{0} \in X$ найдутся окрестности $V_{\varepsilon}^{i}\left(x_{0}\right), i=1, \ldots, m$, такие что множества $S(x) \cap B_{\varepsilon / 2}\left(y^{i}\right)$ будут непустыми для любого $x \in V_{\varepsilon}^{i}\left(x_{0}\right), i=1, \ldots, m$. Выбирая в качестве окрестности точки $x_{0}$ множество $V_{\varepsilon}\left(x_{0}\right)=\bigcap_{i=1}^{m} V_{\varepsilon}^{i}\left(x_{0}\right)$, получаем, что для любого $x \in V_{\varepsilon}\left(x_{0}\right)$ найдутся точки $\hat{y}^{i}(\varepsilon, x) \in S(x)$, такие что $\rho\left(y^{i}, \hat{y}^{i}(\varepsilon, x)\right)<\varepsilon / 2$ для $i=1, \ldots, m$. Положим теперь $Q_{\varepsilon}^{x}=\sum_{i=1}^{m} q_{i} \delta_{\hat{y}^{i}(\varepsilon, x)}$; тогда

$$
\left\|Q_{\varepsilon}-Q_{\varepsilon}^{x}\right\|_{D} \leq \sum_{i=1}^{m} q_{i}\left\|\delta_{y^{i}}-\delta_{\hat{y}^{i}(\varepsilon, x)}\right\|_{D} \leq \sum_{i=1}^{m} q_{i} \rho\left(y^{i}, \hat{y}^{i}(\varepsilon, x)\right)<\varepsilon / 2 .
$$

Таким образом, $\left\|Q_{0}-Q_{\varepsilon}^{x}\right\|_{D} \leq\left\|Q_{0}-Q_{\varepsilon}\right\|_{D}+\left\|Q_{\varepsilon}-Q_{\varepsilon}^{x}\right\|_{D}<\varepsilon$, а значит многозначное отображение $x \mapsto \mathcal{N}(x)$ является $h$-полунепрерывным снизу (для метрики, порожденной нормой Дадли в пространстве $\mathcal{P}(Y))$.

Чтобы убедиться в замкнутости $\mathcal{N}(x)$, достаточно заметить, что $Q \in \mathcal{N}(x)$ равносильно $Q\left((S(x))^{c}\right)=0$; по теореме А.Д. Александрова для слабо сходящейся последовательности $Q_{n} \in$ $\mathcal{N}(x)$ к мере $Q^{*}$ имеем $0=\liminf _{n \rightarrow \infty} Q_{n}\left((S(x))^{c}\right) \geq Q^{*}\left((S(x))^{c}\right)$, т. е. $Q^{*} \in \mathcal{N}(x)$. Выпуклость $\mathcal{N}(x)$ очевидна.

\footnotetext{
${ }^{15}$ Если $X$ - топологическое пространство, $(Y, \rho)$ - метрическое пространство, то многозначное отображение $F: X \mapsto 2^{Y} \backslash\{\varnothing\}$ называется $h$-полунепрерывным снизу в точке $x_{0} \in X$, если $e\left(F\left(x_{0}\right), F(x)\right)$, отклонение Помпею $F\left(x_{0}\right)$ от $F(x)$, непрерывно в точке $x_{0}$. Здесь $e(A, B)=\sup \{\rho(a, B): a \in A\}$, $\rho(x, A)=\inf \{\rho(x, y): y \in A\}$ (см., например,[7, определение 2.60]).

${ }^{16}$ Многозначное отображение $F: X \mapsto 2^{Y} \backslash\{\varnothing\}$ полунепрерывно снизу в точке $x_{0} \in X$, если для любого открытого множества $G \subseteq Y$, такого что $G \cap F\left(x_{0}\right) \neq \varnothing$, найдется окрестность $W_{x_{0}}$ точки $x_{0}$, такая что для всех $x \in W_{x_{0}}$ имеет место $G \cap F(x) \neq \varnothing$.
} 


\section{2. Существование феллеровских переходных ядер}

Предложение 1 из разд. 1 устанавливает необходимое условие феллеровости ядра $Q-$ полунепрерывность снизу многозначного отображения $x \mapsto \operatorname{supp}(Q(x, \cdot))$. Несколько усиливая требования (по сравнению с предыдущим разделом статьи) к пространствам $X$ и $Y$, получаем два утверждения, которые устанавливают в определенном смысле обратные утверждения.

Теорема 1. Пусть $X$ - нормальное счетно паракомпактное пространство ${ }^{17}$ u $Y$ польское пространство ${ }^{18}$, снабженные борелевскими $\sigma$-алгебрами $\mathcal{B}_{X}$ и $\mathcal{B}_{Y}$ соответственно. Если $S: X \mapsto 2^{Y}$ многозначное отображение, принимающее непустые замкнутые значения, полунепрерьвно снизу, то найдется феллеровское переходное ядро $Q=Q(x, B), x \in X$, $B \in \mathcal{B}_{Y}$, maкое что $\operatorname{supp}(Q(x, \cdot)) \subseteq S(x)$.

Д о к а з а т е л ь с т в о. Рассмотрим пополнение $\tilde{\mathcal{M}}(Y)$ пространства зарядов $\mathcal{M}(Y)$, являющееся банаховым пространством относительно нормы Дадли, причем $\mathcal{M}(Y)$ отображается в $\tilde{\mathcal{M}}(Y)$ при помощи изометрического линейного оператора (так что можно говорить о вложении пространства $\mathcal{M}(Y)$ в пространство $\tilde{\mathcal{M}}(Y)$ - как всюду плотного множества). Это пополнение однозначно определено с точностью до изометрического линейного изоморфизма (см., например, [18, теоремы 2.5.2 и 2.5.4]). По теореме 1.11 из классической работы Ю. В. Прохорова [19] пространство вероятностных мер $\mathcal{P}(Y)$ со слабой топологией метризуемо при помощи метрики $d_{L P}$ Леви - Прохорова и является польским. Из этой теоремы, а также из следствий 2 и 3 [20, разд. 2], в соответствии с которыми имеют место неравенства

$$
\begin{aligned}
\left\|Q_{1}-Q_{2}\right\|_{D} & \leq 2 d_{L P}\left(Q_{1}, Q_{2}\right), \\
d_{L P}\left(Q_{1}, Q_{2}\right) & \leq \sqrt{\frac{3}{2}\left\|Q_{1}-Q_{2}\right\|_{D}}
\end{aligned}
$$

для любых $Q_{1}$ и $Q_{2}$ из $\mathcal{P}(Y)$, вытекает замкнутость $\mathcal{P}(Y)$ в $\tilde{\mathcal{M}}(Y)$ (в силу полноты $\mathcal{P}(Y)$ в метрике Дадли). Поэтому замкнутые по норме Дадли подмножеств $\mathcal{P}(Y)$ будут также замкнутыми в $\tilde{\mathcal{M}}(Y)$.

В силу предложения 3 заметим, что многозначное отображение $\mathcal{N}: X \mapsto \mathcal{P}(Y)$, определяемое соотношением (1.1), является $h$-полунепрерывным снизу, а значит и полунепрерывным снизу (см., например, [7, предложение 2.66]). Полунепрерывность снизу $\mathcal{N}$ в пространстве $\tilde{\mathcal{M}}(Y)$ равносильна тому, что для любого замкнутого множества $\mathcal{J} \subseteq \tilde{\mathcal{M}}(Y)$ множество точек $\{x \in X: \mathcal{N}(x) \subseteq \mathcal{J}\}$ замкнуто в пространстве $\tilde{\mathcal{M}}(Y)$. Поскольку $\mathcal{N}(x) \subseteq \mathcal{P}(Y)$, а $\mathcal{P}(Y)$ замкнуто в $\tilde{\mathcal{M}}(Y)$, то

$$
\{x \in X: \mathcal{N}(x) \subseteq \mathcal{J}\}=\{x \in X: \mathcal{N}(x) \subseteq \mathcal{J} \cap \mathcal{P}(Y)\}
$$

где $\mathcal{J} \cap \mathcal{P}(Y)$ - замкнутое подмножество $\mathcal{P}(Y)$, так что полунепрерывное снизу в пространстве $\mathcal{P}(Y)$ отображение $\mathcal{N}$ будет также полунепрерывным снизу и в пространстве $\tilde{\mathcal{M}}(Y)$.

Далее, $\tilde{\mathcal{M}}(Y)$ является сепарабельным, поскольку таковым является $\mathcal{M}(Y)$. Сепарабельность $\tilde{\mathcal{M}}(Y)$ проверяется достаточно легко. Действительно, используя разложение Жордана, конечный заряд $\mu$ единственным образом представим в виде разности взаимно сингулярных (неотрицательных) конечных мер $\mu^{+}$и $\mu^{-}$, т. е. $\mu=\mu^{+}-\mu^{-}$(см., например, [16, разд. 1, §5]). Любую конечную меру $\nu$ можно приблизить мерами вида $r_{n} \pi$, где $\pi \in \mathcal{P}(Y)$, а $r_{n} \geq 0-$ рациональные числа, такие что при $n \rightarrow \infty$ имеет место сходимость $r_{n} \rightarrow\|\nu\|_{D}=\|\nu\|_{T V}=\nu(Y)^{19}$.

\footnotetext{
${ }^{17}$ См. определения, например, в книге $[17$, разд. 1.5 , гл. $1 ;$ разд. 5.2, гл. 5]: счетная паракомпактность топологического пространства означает, что в любое его счетное открытое покрытие можно вписать локально конечное открытое покрытие.

${ }^{18}$ Полное сепарабельное метрическое пространство.

${ }_{19}^{19}$ десь $\|\mu\|_{T V}-$ норма полной вариации заряда $\mu$ на пространстве $(Y, \mathcal{B})$, т. е. $\|\mu\|_{T V}=\mu^{+}(Y)+$ $\mu^{-}(Y)$; в общем случае (знакопеременном), очевидно, $\|\mu\|_{D} \leq\|\mu\|_{T V}$.
} 
В то же время $\mathcal{P}(Y)$ сепарабельно; пусть $\mathcal{P}^{\prime}-$ счетное всюду плотное в $\mathcal{P}(Y)$ множество. Для конечной меры $\nu \neq 0$ положим $\pi=\frac{1}{\|\nu\|_{D}} \nu$, и пусть $\pi_{n} \in \mathcal{P}^{\prime}, \pi_{n} \rightarrow \pi$; тогда

$$
\left\|r_{n} \pi_{n}-\nu\right\|_{D} \leq\left|r_{n}-\|\nu\|_{D}\right|+\|\nu\|_{D}\left\|\pi_{n}-\pi\right\|_{D} \rightarrow 0 .
$$

Таким образом, с учетом предложения 1 многозначное отображение $\mathcal{N}$ представляет собой полунепрерывное снизу многозначное отображение, принимающее непустые выпуклые замкнутые значения, содержащиеся в замкнутом выпуклом подмножестве $\mathcal{P}(Y)$ сепарабельного банахова пространства $\tilde{\mathcal{M}}(Y)$, поэтому применима теорема $3.1^{\prime \prime}$ из классической работы Майкла [4], в соответствии с которой $\mathcal{N}$ имеет непрерывную ветвь (селектор) $x \mapsto Q(x, \cdot) \in \mathcal{N}(x)$. Для завершения доказательства достаточно сослаться на предложение 1.

Незначительное усиление требований к топологическому пространству $X$ позволяет получить следующее утверждение.

Теорема 2. Пусть $X-$ совершенно нормальное пространство $0^{20}$ и $Y$ - польское пространство, снабженные борелевскими б-алгебрами $\mathcal{B}_{X}$ и $\mathcal{B}_{Y}$ соответственно. Eсли $S: X \mapsto 2^{Y}$ многозначное отобраэсение, принимающее непустые замкнутые значения, полунепрерывно снизу, то найдется феллеровское переходное ядро $Q=Q(x, B), x \in X, B \in \mathcal{B}_{Y}$, такое что $\operatorname{supp}(Q(x, \cdot))=S(x)$.

Д о к а з а т е л ь с т в о. Поскольку многозначное отображение $\mathcal{N}: X \mapsto \mathcal{P}(Y)$, определяемое соотношением (1.1), представляет собой полунепрерывное снизу многозначное отображение, принимающее непустые выпуклые замкнутые значения, содержащиеся в замкнутом выпуклом подмножестве $\mathcal{P}(Y)$ сепарабельного банахова пространства $\tilde{\mathcal{M}}(Y)$, пополнения пространства конечных мер $\mathcal{M}(Y)$, применима лемма 5.2 из классической работы Майкла [4], в соответствии с которой найдется счетное семейство непрерывных ветвей (селекторов) $x \mapsto Q_{k}(x, \cdot) \in \mathcal{N}(x), x \in X, k=1,2, \ldots$, таких что для любого $x \in X$ множество мер $\left\{Q_{k}(x, \cdot), k=1,2, \ldots\right\}$ плотно в $\mathcal{N}(x)$. Пусть $Q_{x} \in \mathcal{N}(x)$ - мера, для которой $\operatorname{supp}\left(Q_{x}\right)=S(x)$; такая мера существует по лемме 3 . В силу плотности в $\mathcal{N}(x)$ множества $\left\{Q_{k}(x, \cdot), k=1,2, \ldots\right\}$ найдется (зависящая от $x$ ) подпоследовательность $Q_{k_{l}}(x, \cdot)$, слабо сходящаяся к $Q_{x} \in \mathcal{N}(x)$. Поэтому

$$
\liminf _{l \rightarrow \infty} Q_{k_{l}}(x, G) \geq Q_{x}(G)>0
$$

для произвольного открытого $G$, такого что $G \cap S(x) \neq \varnothing$. Положим

$$
Q^{*}(x, \cdot)=\sum_{k=1}^{\infty} q_{k} Q_{k}(x, \cdot),
$$

где $q_{k}>0, \sum_{k=1}^{\infty} q_{k}=1$. Из $(2.1)$ следует, что $\operatorname{supp}\left(Q^{*}(x, \cdot)\right)=S(x)$ для любого $x \in X$. При этом функция $x \mapsto Q^{*}(x, \cdot)$ непрерывна в слабой топологии, поскольку для ограниченной непрерывной функции $f$ ряд непрерывных функций

$$
x \mapsto \sum_{k=1}^{\infty} q_{k} \int f(y) Q_{k}(x, d y)
$$

сходится равномерно:

$$
\left|\sum_{k=1}^{n} q_{k} \int f(y) Q_{k}(x, d y)-\int f(y) Q^{*}(x, d y)\right|=\left|\sum_{k=n}^{\infty} q_{k} \int f(y) Q_{k}(x, d y)\right| \leq\|f\|_{\infty} \sum_{k=n}^{\infty} q_{k} .
$$

\footnotetext{
${ }^{20}$ См. определение, например, в книге [17, разд. 1.5, гл. 1]: топологическое пространство называют совершенно нормальным, если оно нормально и любое замкнутое множество является множеством типа $G_{\delta}$, т. е. представимо в виде пересечения счетного числа открытых множеств. Совершенно нормальное пространство является счетно паракомпактным (см. например, [17, следствие 5.2.5]).
} 
Таким образом, с учетом предложения $1 Q^{*}$ является феллеровским переходным ядром.

3 а м е ч а н и е.

1) Для сепарабельности пространства $\mathcal{P}(Y)$ полнота $Y$ не требуется, достаточно, чтобы $Y$ было сепарабельным метрическим пространством. В $\mathcal{P}(Y)$, например, счетным всюду плотным множеством будет множество мер с конечными носителями, содержащимися в счетном всюду плотном подмножестве $Y$, представимыми в виде выпуклой комбинации мер Дирака (вероятностных мер, сосредоточенных в одной точке) с рациональными коэффициентами (см., например, [21, предложение 2.5.40 $]^{21}$ ).

2) Условия на топологическое пространство $X$ в теоремах 1 и 2 выполняются, если $X-$ метрическое пространство.

Автор выражает благодарность В. И. Тариеладзе за обсуждение и анонимному рецензенту за ценные замечания, способствовавшие существенному улучшению изложения.

\section{СПИСОК ЛИТЕРАТУРЫ}

1. Смирнов С.Н. Феллеровский процесс // Математическая энциклопедия / ред. И. М. Виноградов. Т. 5. М.: Изд-во "Советская энциклопедия", 1985. С. 603-604.

2. Куржанский А.Б. Управление и наблюдение в условиях неопределенности. М.: Наука, 1977. $392 \mathrm{c.}$

3. Tulcea I.C. Mesures dans les espaces produits // Atti Accad. Naz. Lincei. Rend. Cl. Sci. Fis. Mat. Nat. 1949. Vol. 8, iss. 7. P. 208-211.

4. Michael E.A. Continuous selections. I // Ann. Math. 1956. Vol. 63, iss. 2. P. 361-382. doi: $10.2307 / 1969615$.

5. Lange K.L. Borel sets of probability measures // Pacific J. Math. 1973. Vol. 48, iss. 1. P. 141-162.

6. Vakhania N.N., Tarieladze V.I., Chobanyan S.A. Probability distributions on Banach spaces. Dordrecht: D. Reidel Publ. Comp., 1987. 482 p.

7. Hu S., Papageorgiou N. Handbook of multivalued analysis. Vol. I: Theory. Berlin: Springer, 1997. 968 p. (Mathematics and Its Appl.; vol. 419).

8. Биллингсли П. Сходимость вероятностных мер. М.: Наука, 1977. 352 с.

9. Alexandroff A.D. Additive set-functions in abstract spaces // Мат. сб. 1943. Т. 13 (55), № 2-3. C. $169-238$.

10. Ash R.B. Real analysis and probability. N Y: Acad. Press., 1972. 494 p.

11. Богачев В.И. Основы теории меры. Т. 2. М.-Ижевск: НИЦ "Регулярная и хаотическая динамика", 2003. $576 \mathrm{c}$.

12. Ширяев А.Н. Вероятность-1. М.: МЦНМО, 2004. 520 с.

13. Weaver N. Lipschitz algebras. Singapore: World Scientific Publishing Co. Pte. Ltd., 1999. 223 p.

14. Hille S.C., Worm D.T.H. Embedding of semigroups of Lipschitz maps into positive linear semigroups on ordered Banach spaces generated by measures // Integr. Equ. Oper. Theory. 2009. Vol. 63, iss. 3. P. $351-371$.

15. Dudley R.M. Real analysis and probability. Cambridge: Cambridge University Press., 2004. 555 p.

16. Колмогоров А.Н., Фомин С.В. Элементы теории функций и функциональный анализ. М.: Наука, 1976. 543 с.

17. Энгелькинг Р. Общая топология. М.: Мир, 1986. 752 с.

18. Jain P.K., Khalil Ahmad, Ahuja Om P. Functional analysis. New Delhi etc.: New Age International, 1995. $326 \mathrm{p}$.

19. Прохоров Ю.В. Сходимость случайных процессов и предельные теоремы теории вероятностей // Теория вероятностей и ее применение. Т. 1, № 2. 1956. С. 177-238.

20. Dudley R.M. Distances of probability measures and random variables // Ann. Math. Statist. 1968. Vol 39, iss 5. P. 1503-1572. doi: 10.1214/aoms/1177698137.

\footnotetext{
${ }^{21} \mathrm{~B}$ формулировке этого предложения авторы забыли упомянуть про рациональность коэффициентов (хотя имели это в виду).
} 
21. Denkowski Z., Migorski S., Papageorgiou N.S. An Introduction to nonlinear analysis: Theory. N Y: Springer Science \& Business Media, 2003. 823 p.

Поступила 13.07.2018

После доработки 16.11.2018

Принята к публикации 19.11.2018

Смирнов Сергей Николаевич

канд. физ.-мат. наук,

Московский государственный университет им. М. В. Ломоносова

г. Москва

e-mail: s.n.smirnov@gmail.com

\section{REFERENCES}

1. Smirnov S.N. Feller Processes. In: Encyclopaedia of mathematics, vol. 10. N Y: Springer, 2012, pp. 483485.

2. Kurzhanskii A.B. Upravlenie $i$ nablyudenie $v$ usloviyakh neopredelennosti [Control and Observation Under the Conditions of Uncertainty]. Moscow, Nauka Publ., 1977, 392 p.

3. Tulcea I.C. Mesures dans les espaces produits. Atti Accad. Naz. Lincei. Rend. Cl. Sci. Fis. Mat. Nat., 1949, vol. 8, no. 7, pp. 208-211.

4. Michael E.A. Continuous selections. I. Ann. Math., 1956, vol. 63, no. 2, pp. 361-382. doi: $10.2307 / 1969615$.

5. Lange K.L. Borel sets of probability measures. Pacific J. Math., 1973, vol. 48, no. 1, pp. 141-162.

6. Vakhania N.N., Tarieladze V.I., Chobanyan S.A. Probability distributions on Banach spaces. Dordrecht, D. Reidel Publishing Company, 1987, 482 p. ISBN: 90-277-2496-2 .

7. Hu S., Papageorgiou N. Handbook of multivalued analysis. Vol. I: Theory. Ser. Math. and Its Appl., vol. 419. Berlin: Springer, 1997, 968 p. ISBN: 0792346823.

8. Billingsley P. Convergence of probability measures. N Y: Wiley, 1968, 253 p. ISBN: 0471072427. Translated to Russian under the title Skhodimost' veroyatnostnykh mer. Moscow, Nauka Publ., 1977, $352 \mathrm{p}$.

9. Alexandroff A.D. Additive set-functions in abstract spaces. Rec. Math. [Mat. Sbornik] N.S., 1943, vol. 13 (55), no. 2-3, pp. 169-238.

10. Ash R.B. Real analysis and probability. N Y: Acad. Press., 1972. 494 p. ISBN: 978-0120652013.

11. Bogachev V.B. Measure theory, Vol. II. N Y: Springer, 2007, 586 p. Original Russian text published in Bogachev V.I., Osnovy teorii mery, vol. 2. M.; Izhevsk: NITs "Regulyarnaya i Khaoticheskaya Dinamika" Publ., 2003, 576 p.

12. Shiryaev A.N. Probability. Ser. Graduate Texts in Math., vol. 95, N Y etc.: Springer-Verlag, 1995, 624 p. ISBN: 0387945490 . Original Russian text (3rd ed.) published in Shiryaev A.N. Veroyatnost'-1. Moscow, Nauka Publ., 2004, 408 p.

13. Weaver N. Lipschitz algebras. Singapur: World Scientic Publishing Co. Pte. Ltd., 1999, 223 p. doi: $10.1142 / 4100$.

14. Hille S.C., Worm D.T.H. Embedding of semigroups of Lipschitz maps into positive linear semigroups on ordered Banach spaces generated by measures. Integr. Equ. Oper. Theory, 2009, vol. 63, no. 3, pp. 351371. doi: 10.1007/s00020-008-1652-z .

15. Dudley R.M. Real analysis and probability. Cambridge: Cambridge University Press, 2004, 555 p. ISBN: 0-511-04208-6.

16. Kolmogorov A.N., Fomin S.V. Elements of the theory of functions and functional analysis. Vol. 1, 2. Mineola, N Y, Dover Publ., 1999, 288 p. ISBN: 0486406830 . Original Russian text (4th ed.) published in Kolmogorov A.N., Fomin S.V. Elementy teorii funktsii i funktsional'nogo analiza. Moscow, Nauka Publ., $1976,543 \mathrm{p}$.

17. Engelking R. General topology. Sigma series in pure mathematics, vol. 6, Berlin: Heldermann Verlag, 1989, 535 p. ISBN: 3885380064 . Translated to Russian inder the title Obshchaya topologiya. Moscow: Mir Publ., 1986, 752 p.

18. Jain P.K., Khalil Ahmad, Ahuja Om P. Functional analysis. New Delhi etc.: New Age International, 1995, 326 p. ISBN: 9788122408010 . 
19. Prokhorov Yu.V. Convergence of random processes and limit theorems in probability theory. Theory Probab. Appl., 1956, vol. 1, no. 2, pp. 157-214. doi: 10.1137/1101016.

20. Dudley R.M. Distances of probability measures and random variables. Ann. Math. Statis., 1968, vol. 39, no. 5, pp. 1563-1572. doi: 10.1214/aoms/1177698137.

21. Denkowski Z., Migorski S., Papageorgiou N.S. An introduction to nonlinear analysis: Theory. N Y: Springer Science \& Business Media, 2003, 823 p. ISBN: 0306474565.

Received July 13, 2018

Revised November 16, 2018

Accepted November 19, 2018

Funding Agency: This study was carried out at the Faculty of Computational Mathematics and Cybernetics of Moscow State University within the project "Optimization Methods in Control Problems for Complex Systems under Available Information" (state registration no. AAAA-A16116021110324-8).

Sergei Nikolaevich Smirnov, Cand. Sci. (Phys.-Math.), Lomonosov Moscow State University, Moscow, 119991 Russia, e-mail: s.n.smirnov@gmail.com . 